\title{
Stool culture: Are you getting value for money?
}

\author{
C A Persaud, S J Eykyn
}

\begin{abstract}
Aims-To assess the best medium for primary isolation of enteric pathogens; to determine the need for a second primary culture medium; and to gather information on stool culture media used in 20 other randomly selected laboratories.

Methods-Specimens were cultured on desoxycholate citrate agar (DCA), Hektoen enteric agar (Hektoen), and xylose lysine deoxycholate agar (XLD). Non-lactose fermenters were screened with Rapidec Z (bio-Mérieux) and identified with API 10S (bio-Mérieux) where appropriate. Shigellas were identified with API 20E (bio-Mérieux) and serology, and salmonellas biochemically and by serology. A telephone survey was carried out to enquire into different culture practices and whether they had been evaluated for cost effectiveness.

Results-The isolation rate of enteric pathogens on primary stool culture media was $97 \%$ on DCA, $88 \%$ on XLD, and $76 \%$ on Hektoen. Seventeen of 18 shigellas grew on DCA, 13 of 18 on XLD, and 14 of 18 on Hektoen. DCA missed one Salmonella, XLD three, and Hektoen 13. XLD and Hektoen both missed Yersinia enterocolitica. The telephone survey revealed a diverse range of both primary and subculture plates. There was little evidence of evaluation of stool media, but firm personal convictions concerning the advantages and disadvantages of each type of medium at each stage of culture. Conclusions-DCA performed best and was the most cost effective of the three media. Neither XLD nor Hektoen were satisfactory as primary culture media because they grew fewer pathogens than DCA.
\end{abstract}

\section{$(\mathcal{F}$ Clin Pathol 1994;47:790-792)}

All microbiologists "know" which medium is best for the isolation of enteric pathogens. We know that Hektoen and XLD are best for shigellas, and that DCA is too inhibitory for these organisms to grow well. Our routine plates for primary stool culture were DCA and Hektoen. Hektoen had been introduced as a second primary culture plate in 1988 , as we "knew" that shigellas grew better on this plate, and also "felt" that we should not rely on one primary plate alone.

Although regional audits have examined various aspects of stool investigation, including media, standard investigations for given clinical details, and sensitivity testing, there is little information comparing actual performance of enteric media. Because stool microbiology is expensive, a study was designed to assess what methods laboratories used and the most cost effective media for stool culture.

\section{Methods}

The stool study took place over two months in 1993 and involved a number of bench staff to reflect routine laboratory practice. By varying bench staff, individual expertise with any particular medium was minimised and the media challenged fully. DCA (Unipath CM35), XLD (Unipath CM469), and Hektoen (bioMérieux 51050) media were made according to the manufacturer's instructions and subjected to our in-house quality control panel of organisms. DCA was stored in the dark at room temperature to prevent the development of the red precipitate (sodium desoxycholate).

Specimens included consecutive routine stools $(\mathrm{n}=853)$, NEQAS stool specimens $(n=2)$, and simulated stools $(n=13)$ Simulated specimens were made by the addition of a 4-6 drops of McFarlane standard 2 or 4 of Salmonella enteritidis, $S$ typhimurium or Shigella sonnei (or a mixture of two) to $1 \mathrm{ml}$ of known negative stool sample. An artificially high number of positive specimens was tested to challenge the media.

Specimens were inoculated on the agar plates with a cotton wool swab to produce a pool, which was streaked out with a loop to give single colonies. A pea-sized amount of specimen was inoculated into Selenite Broth (Unipath). Plates and enrichment broth were incubated at $37^{\circ} \mathrm{C}$ for 18 hours. Non-lactose fermenters were investigated with Rapidec $Z$ and "positive" organisms with this system identified with API 10S. The identity of shigellas was confirmed with API 20E and serology and that of salmonellas by serology. (The amount of work on each plate was monitored by recording screen test and identification test results).

To obtain a perspective of laboratory practices in stool culture, a telephone survey of 20 random laboratories in the United Kingdom was undertaken by CAP. Each laboratory was also asked whether the media had been evaluated for cost effectiveness. (These included NHS, PHLS, and private microbiology laboratories).

\section{Results}

STOOL CULTURE STUDY

A total of 74 pathogens was isolated from the primary plates: 55 salmonellas (38 $S$ enteritidis, $11 S$ typhimurium, three $S$ hadar, 
one $S$ agona, one $S$ livingstone, one $S$ saintpaul), 18 shigellas (12 $S$ sonnei, three $S$ flexneri, two $S$ boydii, one $S$ dysenteriae) and $Y$ enterocolitica.

DCA yielded 72 of 74 (97\%) pathogens, but failed to isolate one $S$ enteritidis and one $S$ sonnei. XLD yielded 65 of 74 (88\%) pathogens, missing three salmonellas, all $S$ enteritidis, five shigellas (three $S$ sonnei, one $S$ flexneri, and one $S$ dysenteriae) and the $Y$ enterocolitica. Hektoen yielded 56 of 74 (76\%) pathogens, missing 13 salmonellas (10 $S$ enteritidis, two $S$ hadar, one $S$ agona), three shigellas (one $S$ sonnei, one $S$ flexneri, one $S$ boydir) and the $Y$ enterocolitica.

We also compared the number of non-lactose fermenting colonies present on each plate and correlated this with pathogens isolated, to assess the number of "false positives" (where a false positive is a non-lactose fermenter, not an enteric pathogen). A total number of 184 non-lactose fermenters were isolated on DCA, 177 on XLD, and 205 on Hektoen. Preliminary screening of non-lactose fermenters using Rapidec $\mathrm{Z}$ left 148 isolates on DCA plates requiring further identification. From these, 65 pathogens were identified. For Hektoen, preliminary screening left 153 isolates requiring further identification. From these, 56 pathogens were identified.

\section{TELEPHONE SURVEY}

The number of plates used for primary culture and subculture in the 20 laboratories surveyed varied widely. Only two of the 20 $(10 \%)$ laboratories used one medium for primary stool culture, $13(65 \%)$ used two, and five $(25 \%)$ used three. Fifteen $(75 \%)$ used only one medium for subculture for their enrichment broth, three (15\%) used two and two $(10 \%)$ three. The types of media used are shown in the table. Very few laboratories had undertaken critical assessment of the media they used, although many senior technical staff held strong convictions concerning the best media. The following were some of the comments offered.

Comparison of stool culture media in the 20 laboratories surveyed

\begin{tabular}{|c|c|c|}
\hline Laboratory & Primary plate(s) & Subculture plate(s) \\
\hline $\begin{array}{l}1 \\
2 \\
3 \\
4 \\
5 \\
6 \\
7 \\
8 \\
9 \\
10 \\
11 \\
12 \\
13 \\
14 \\
15 \\
16 \\
17 \\
18 \\
19 \\
20\end{array}$ & $\begin{array}{l}\text { DCA, XLD, W and B } \\
\text { DCA, XLD } \\
\text { DCA(H) } \\
\text { DCA(H), XLD } \\
\text { DCA(H), XLD } \\
\text { DCA(H), XLD, Sorb, BA } \\
\text { DCA, MAC } \\
\text { DCLS } \\
\text { DCA, XLD, MAC } \\
\text { DCLS, XID } \\
\text { DCLS, MAC } \\
\text { DCA(H), MAC } \\
\text { DCLS, XID } \\
\text { DCLS, MAC } \\
\text { DCA, XLD } \\
\text { XLD, HEK, AERO } \\
\text { DCA(H), MAC } \\
\text { DCLS, MAC } \\
\text { XLD, HEK } \\
\text { DCA, W and B }\end{array}$ & $\begin{array}{l}\text { DCA, XLD, W and B } \\
\text { DCA } \\
\text { DCA(H) } \\
\text { DCA(H), XLD } \\
\text { DCA(H), XLD } \\
\text { DCA(H), XLD } \\
\text { DCA } \\
\text { XLD } \\
\text { XLD } \\
\text { DCLS } \\
\text { DCLS } \\
\text { DCA(H) } \\
\text { DCLS, XLD } \\
\text { DCLS } \\
\text { DCA } \\
\text { XLD } \\
\text { DCA } \\
\text { DCLS } \\
\text { XLD } \\
\text { DCA, W and B }\end{array}$ \\
\hline
\end{tabular}

AERO = Aeromonas selective agar; $B A=$ blood agar; $D C A=$ desoxycholate citrate agar; DCA $(\mathrm{H})=\mathrm{DCA}-$ Hynes's modification; DCLS = DCA modified to contain sucrose; HEK $=$
Hektoen; MAC = MacConkey agar; Sorb = sorbitol MacConkey; W and B = Wilson and Blair.
"We feel that non-lactose fermenters are easier to see on XLD"

"Hektoen is best, but we can't afford it!"

"We feel that Wilson and Blair medium grows more scanty salmonellas at 24 hours."

"It is easier to spot non-lactose fermenters on XLD."

"We have used XLD and MacConkey in the past and missed shigellas, so we added DCA."

"XLD is clearer to read and good for mixtures."

"We are aware of costings, but they are not important[...]"

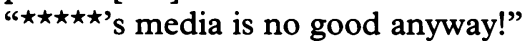

\section{Discussion}

From analysis of these results and within the limitations of this study, DCA was the best medium for primary stool culture. All clinical isolates in this study were recovered from a DCA primary plate and subculture on Selenite Broth (Unipath) to DCA. DCA failed to isolate $S$ sonnei from a simulated (internal QC) sample containing both $S$ sonnei and $S$ enteritidis. Such mixtures are known to cause difficulties for stool cultures, yet their actual incidence is very low. In this study, only two mixtures were encountered in nearly 900 specimens (simulated specimen and a NEQAS sample distribution No 787), despite the use of three primary plates. We have never missed mixtures of enteric pathogens in NEQAS specimens, and feel that this failure to isolate a single shigella from a mixture does not justify the routine use of more than one plate.

XLD performed second best and Hektoen worst. It was not possible to say whether failure to isolate a pathogen on a particular agar was due to an overgrowth of other flora, or inability of the medium to support the growth of the pathogen.

A number of evaluations of enteric media have been done in the past. ${ }^{1-3}$ Isenburg compared XID, Hektoen, and SalmonellaShigella agar and found the former two superior for shigellas. ${ }^{1}$ Taylor compared XLD, Hektoen, DCA, Salmonella-Shigella agar and Eosin Methylene Blue agar and found that XLD and Hektoen agar produced better growth of shigellas. ${ }^{2}$ There are no recent comparisons of DCA, DCLS (desoxycholate citrate lactose sucrose agar), and XLD, yet these media constitute $77 \%$ of media used in the laboratories surveyed. This is particularly relevant as manufacturers are regularly modifying the constituents of the media to improve performance.

Most laboratories use a second medium for the same reasons that we used to. Tradition dictates the inclusion of an extra plate for shigellas. Our study suggests it is unnecessary and neither Hektoen or XLD were useful adjuncts to complement our stool culture methods. In fact, Hektoen had more false positives than other media. This supports the findings of Taylor et al. ${ }^{2}$ 


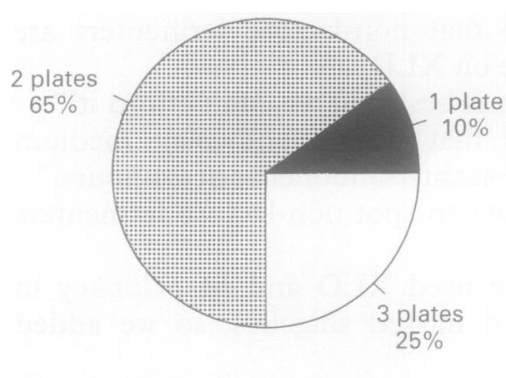

Primary culture

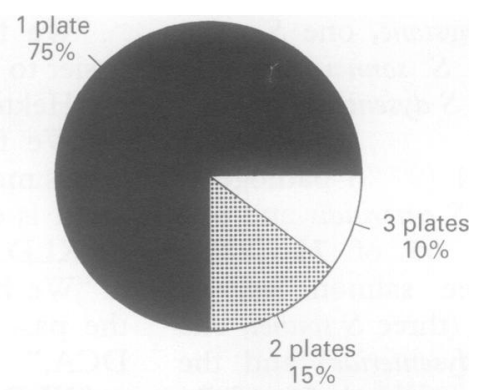

Post-enrichment broth subculture plates

Comparison of the number of plates used in routine stool culture in 20 laboratories.

Our own (and regional) audit studies have shown that stool cultures are the most expensive investigations of routine microbiology. We estimate that excluding Hektoen has saved us approximately $£ 7000 /$ year.

We rely heavily on tradition, "feeling" that we should use more than one plate, and "knowing" that we are happy with our choice. It may be argued that NEQAS provides sufficient quality control of media (seven salmonella/shigella isolates in 1992), but inevitably NEQAS specimens are given priority treatment, usually with more cultures and time than routine specimens, which defeats the objective. If a NEQAS enteric pathogen is missed a repeat sample can be retested, but the information relating to agar type and manufacturer is not collated. Perhaps laboratories have opted for the easiest option when missing a NEQAS isolate-to add another plate.

Recent development of enteric media has focused on the ability to produce characteristic "crimson coloured" salmonella colonies, and thus avoid costly screening of non-lactose fermenters. Rambach produced a biochemically specific medium on which salmonellas have a classic appearance, and no false positives were found, ${ }^{4}$ and Grunewald et al found the same, ${ }^{5}$ although $S$ typhi and $S$ paratyphi do not produce classic colonies (due to biochemical differences). The advantage of this medium is its specificity, but it is expensive and can not be

used as a single primary plate for stool culture because it is designed only for salmonellas. Most of the laboratories surveyed (90\%) used at least two primary culture plates, with DCA (including Hynes' modification), DCLS, and XLD the most popular media.

It is widely accepted that the formula of a given agar differs among manufacturers. For this reason the poor performance of Hektoen agar in our study does not necessarily mean that Hektoen from different sources will perform poorly. Similarly, it does not mean that DCA, regardless of source, will be the "best" performer. Our study used the same batch (power) of agar for DCA and Hektoen and two different batches of XLD. We cannot comment on the effects of interbatch variation of XLD, but feel that the stringent quality assurance that manufacturers use whereby batches of inferior quality are rejected, ensures a high degree of consistency. It would be interesting to see published comparisons of the performance of the same agar from different manufacturers.

Every method for stool culture will miss a small number of pathogens. As our NEQAS results for stool culture have been $100 \%$ (without relying on a Hektoen plate) over the past two years, our current methods seem to be satisfactory.

We thank all the laboratories who provided the information in the telephone survey, St Thomas's Hospital microbiology MLSOs, and Dr Dybowski for their assistance.

1 Isenberg Henry D, Kominos S, Siegel Marie. Isolation of Salmonellae and Shigellae from an artificial mixture of Salmonellae and Shigellae from an artificial

2 Taylor Welton I, Schelhart Dorothy. Isolation of Shigellae VIII. Comparison of xylose lysine deoxycholate agar, Hektoen enteric agar, salmonella-shigella agar, and eosin methylene blue agar with stool specimens. Appl Microbiol 1971;21:32-7.

3 Pollock Helen $M$, Dahlgren Bonnie J. Clinical evaluation of enteric media in the primary isolation of Salmonella and Shigella. Appl Microbiol 1974;27:197-201.

4 Rambach A. New plate medium for facilitated differentiation of Salmonella spp from Proteus spp and other enteric bacteria. Appl Environmental Microbiol 1990; 56:301-3.

5 Grunewald R, Henderson R, Yappow S. Use of Rambach propylene glycol containing agar for identification of Salmonella spp. $\mathcal{F}$ Clin Microbiol 1991;29:2354-6. 